\title{
Pronóstico de las exportaciones del cacao Ecuatoriano para el 2018 con el uso de modelos de series de tiempo
}

\section{Forecast of exports of Ecuadorian cocoa for 2018 with the use of time series models}

Hugo Renán Ruiz López

Universidad Internacional del Ecuador, Ecuador

Autor por correspondencia: huruizlo@uide.edu.ec

Fecha de recepción: 25 de Febrero de 2018 - Fecha de aceptación: 25 de Junio de 2018

\begin{abstract}
Resumen
Gran parte de las decisiones que se toman en las empresas públicas o privadas, se hacen a partir de pronósticos y de error o incertidumbre asociado con estos. El presente trabajo se realiza un pronóstico de las exportaciones de cacao ecuatoriano para el 2018, se toman como referencia las exportaciones de los últimos ocho años. Se utilizaron métodos de pronósticos para el corto plazo como son las medias móviles y suavización exponencial, adicionalmente se realizó un pronóstico trimestral para el 2018 con el método multiplicativo estacional.
\end{abstract}

Palabras Claves: pronósticos; series de tiempo; medias móviles; suavización exponencial; exportaciones; cacao

\begin{abstract}
A large part of the decisions made in public or private companies are made based on forecasts and the error or uncertainty associated with them. The present work is a forecast of Ecuadorian cocoa exports for 2018, exports of the last eight years are taken as reference. We used forecasting methods for the short term such as moving averages and exponential smoothing, additionally a quarterly forecast was made for 2018 with the seasonal multiplicative method.
\end{abstract}

Key Words: forecasts; time series; moving averages; exponential smoothing; exports; cocoa 


\section{Introducción}

Un aspecto esencial de la administración de cualquier organización es la planeación del futuro. En efecto, el éxito a largo plazo de una organización depende de cuán bien la gerencia anticipa el futuro y elabora estrategias apropiadas. (Anderson, Sweeney, Williams, Camm, \& Martin, 2011).

Como se menciona, el uso de pronósticos de la demanda es necesario para una buena administración de cualquiera empresa, ya que la planeación y control de las actividades logísticas requieren estimaciones precisas de los volúmenes de productos y servicios que serán manejados, estas estimaciones se realizan con pronósticos y estos a su vez son generados en varios departamentos, como son mercadotecnia, planeación de la producción, logística u otros. (Enríquez, 2009).

No solo los pronósticos son cada vez más importantes, si no que los modelos cuantitativos desempeñan un papel cada vez más crucial en la función de pronosticar. Claramente existe un crecimiento continuo en el uso de modelos de pronósticos cuantitativos en todos los niveles en la industria y en el gobierno. (Eppen, Gould, Schmidt, Moore, \& Weatherford, 2000).

En el presente trabajo intenta modelar las exportaciones ecuatorianas de cacao aplicando modelos de series de tiempo, los datos fueron tomados del Banco Central del Ecuador de los periodos 2013 al 2017.

\section{El cacao en el Ecuador}

Ecuador es el cuarto exportador mundial de cacao en grano y lidera la producción de cacao fino de aroma, con una participación del $63 \%$ a escala global, según lo detallan los balances del Banco Central del Ecuador (BCE) en donde se reflejan los principales productos nacionales de exportación (no petroleros) evidenciando que los primeros lugares los ocupa el banano, camarón y cacao. (http://www.ecuadorinmediato.com, 2018).

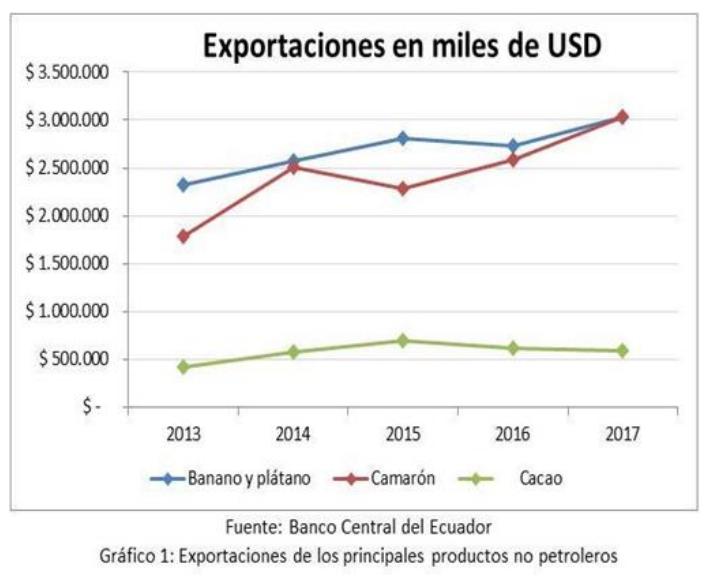

El cacao es una fruta tropical, sus cultivos se encuentran mayoritariamente en la costa y parta de la Amazonía. Es un árbol con flores pequeñas que se observan en las ramas y producen 
una mazorca que contiene granos cubiertos de una pulpa rica en azúcar. La producción de cacao se concentra principalmente en las provincias de Los Ríos, Guayas, Manabí y Sucumbíos. (http://www.revistalideres.ec)

En el país se cultivan dos tipos de cacao: el Cacao CCN-51 y el denominado Cacao Nacional. Es un Cacao Fino de Aroma conocido como 'Arriba', desde la época colonial. Ecuador es el país con la mayor participación en este segmento del mercado mundial (un 63\% de acuerdo con las estadísticas de ProEcuador). (http://www.revistalideres.ec).

\section{Modelos de series de tiempo}

Una serie de tiempo se le denomina a cualquier variable que conste de datos reunidos, registrados u observados sobre incrementos sucesivos de tiempo. Por lo tanto, se concluye que es una secuencia ordenada de observaciones sobre una variable en particular. (Murillo, Trejos, \& Carvajal, 2003).

Si los datos históricos se rigen a valores pasados de la variable que tratamos de pronosticar, el procedimiento de elaboración de pronósticos se llama método de serie de tiempo. El objetivo de los métodos de serie de tiempo es descubrir patrones en los datos históricos y luego extrapolarlos hacia el futuro. (Anderson, Sweeney, Williams, Camm, \& Martin, 2011).

\section{Componentes de una serie}

El patrón o comportamiento de los datos en una serie de tiempo tiene varios componentes. El supuesto es que cuatro componentes separados: tendencia, cíclico, estacional e irregular, se combinen para proporcionar valores específicos de la serie de tiempo.

- Tendencia: Es una serie de tiempo que contiene cambios o movimientos graduales hacía valores de crecimiento o declinación en un periodo prolongado de tiempo.

- Estacional: Se define como estacionalidad una serie de tiempo al interés que muestran los consumidores de un producto o servicio durante una determinada época de un año. Por ejemplo, las ventas de los juguetes en época de navidad, la venta de los paraguas en época de invierno.

- Ciclo: El ciclo se lo define como una secuencia de puntos por encima y por debajo de la línea de tendencia que dura más de un año.

- Irregular: Es el factor residual que incluye las desviaciones de los valores de serie de tiempo reales de aquellos esperados, es decir este componente representa la variabilidad aleatoria de la serie de tiempo.

\section{Métodos de suavización}

Los métodos de suavización son fáciles de usar y por lo general proporcionan un alto nivel de precisión para pronósticos de corto alcance para un siguiente periodo. Estos modelos 
están basados en la suposición de que el patrón de la variable dependiente en el pasado habrá de continuar en el futuro.

- Promedios Móviles Simples: Utiliza el promedio de los n valores más recientes en la serie de tiempo. Este método resulta más útil cuando la demanda no tiene tendencias pronunciadas ni influencias estacionales.

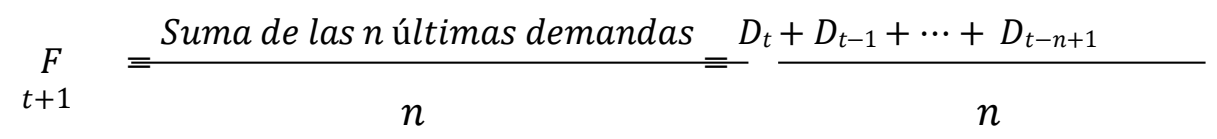

Donde $D t=$ demanda real en el periodo $t$

$F t+1=$ pronóstico para el perido $t+1$

$n=$ número de periodos

- Promedios Móviles Ponderados: En el método de promedio móvil simple, todas las $\mathrm{n}$ demandas tienen la misma ponderación en el promedio, mientras que el promedio móvil ponderado, cada una de las demandas históricas que intervienen en el promedio puede tener su propia ponderación. En la mayoría de los casos, la observación más reciente recibe el mayor peso, y el peso disminuye para valores de datos más antiguos. El resultado de la suma de las ponderaciones es uno.

$$
F t+1=0.7 D t+0.2 D t-1+0.1 D t-2
$$

- Suavización Exponencial Simple: Es un método que permite calcular el promedio de una serie de tiempo, se asignan a las demandas recientes mayor ponderación que a las demandas anteriores.

Es un método que se utiliza más a menudo por su simpleza y por la reducida cantidad de datos que necesita. La suavización requiere solamente tres tipos de datos: el pronóstico del último periodo, la demanda de ese periodo y un parámetro suavizador alfa $\alpha$, cuyo valor fluctúa entre 0 y 1.

$$
\begin{gathered}
F t+1=\propto(\text { Demanda para este perido })+(1-\propto)(\text { Pronóstico del último perido }) \\
F t+1=\propto(D t)+(1-\propto)(F t) \\
F t+1=F t+\propto(D t-F)
\end{gathered}
$$

- Suavización Exponencial Ajustada a la Tendencia: El método para estimar una tendencia es similar al que se emplea para estimar el promedio de la demanda con suavización simple, en este procedimiento se incorpora una tendencia a un pronóstico suavizado exponencialmente. El método consiste en que las estimaciones para el promedio y la tendencia son suavizadas, por ello ahora se requiere dos constantes de suavización llamadas alfa $\alpha$ y Beta $\beta$.

$A t=\propto($ demanda en este periodo $)+(1-\propto)($ Promedio + estimación de la tendencia del último perido)

$A t=\propto D t+(1-\propto)(A t-1+T t-1)$

$T t=($ Promedio de este periodo - Promedio periodo anterior $)+(1-\beta)($ Tendencia último peridod) 
$T t=(A t-A t-1)+(1-\beta) T t-1$

Dónde:

$$
F t+1=A t+T t
$$

$A t=$ promedio exponencialmente suavizado de la serie en el periodo $t$

$T t=$ promedio exponencialmente suavizado de la tendecia en el periodo $t$

$\propto=$ parámetro de suavización exponencial para el promedio, con un valor entre 0 y 1

$\beta=$ parámetro de suavización exponencial para la tendencia, con un valor entre 0 y 1

$F t+1=$ pronóstico para el periodo $t+1$

\section{Patrones estacionales}

En muchas empresas u organizaciones públicas o privadas suelen tener una demanda del tipo estacional. Como se mencionó anteriormente los patrones estacionales son movimientos ascendentes o descendente de la demanda que se repiten con frecuencia, estos son medidos en un periodo de tiempo no mayor a un año (horas, días, semanas, meses o trimestres). Estos periodos de tiempo se llaman estaciones.

- Método Estacional Multiplicativo: Si el comportamiento histórico de la demanda tiene una marcada forma estacional, una manera práctica de realizar el pronóstico es utilizando los índices estacionales. Este método consiste en calcular los factores estacionales y luego multiplicar por una estimación de la demanda del promedio, de esta manera obtenemos un pronóstico estacional.

Este método consta de los siguientes pasos:

- Para cada año, calcule la demanda promedio por estación.

- Para cada año, divida la demanda real correspondiente a una estación entre la demanda promedio por estación. Este resultado será un índice estacional.

- Calcular el índice estacional promedio para cada estación.

- Calcular el pronóstico de cada estación para el siguiente año.

Error de pronóstico: Los pronósticos casi siempre contienen errores, estos errores se pueden clasificar en dos formas:

- Error de sesgo: se dan como resultado de equivocaciones sistemáticas, casi siempre esos errores se dan por ignorar o no estimar correctamente ciertos patrones de demanda.

- Error aleatorio: es el resultado de factores impredecibles (huelgas, paros, el tiempo) que obligan a los pronósticos a desviarse de la demanda real.

Siempre se intenta minimizar los errores seleccionando el método que más se ajuste a la serie, pero es imposible eliminar el error de los pronósticos. El error del pronóstico es la diferencia entre el valor pronosticado y el valor real de la demanda para un determinado periodo, de acuerdo a la siguiente fórmula:

$$
E t=D t-F t
$$


Dónde:

$E t=$ error de pronóstico para el perido $t$

$D t=$ demanda real para el perido $t$

$F t=$ pronóstico para el periodo $t$

A veces es preferible medir el error del pronóstico en un periodo de tiempo largo que un periodo específico, por esta razón se suman los errores de pronóstico. Esta suma acumulativa de errores se la conoce como CFE.

$$
C F E=\sum E t
$$

El error de pronósticos promedio está dado por:

$$
E=\underline{C F E}
$$

$n$

La desviación media absoluta MAD que mide la dispersión de los errores de pronóstico, está dada por:

$$
M A D=\frac{\sum|E t|}{n}
$$

Mientras que el cuadro del error medio (MSE) mide la dispersión de los errores de pronóstico:

$$
M S E=\frac{\sum E 2 t}{n}
$$

\section{Pronóstico de exportaciones de cacao ecuatoriano para el 2018}

Las exportaciones de cacao del Ecuador han tenido un crecimiento casi continuo desde el año 2010 hasta el 2015, luego de este periodo se ha visto un decrecimiento en los dos últimos años, en parte por la baja del precio del mismo derivado por el excedente de la producción. Las exportaciones registradas en el 2017 fueron de \$ 588,4 millones de dólares (BCE).

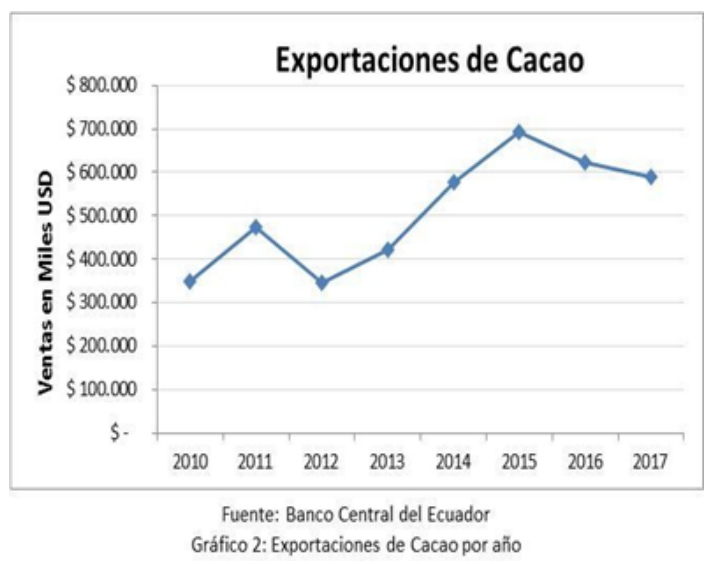

- Promedios Móviles Simple: Nuestro estudio comienza utilizando los promedios móviles de dos y tres periodos para calcular el pronóstico, los resultados fueron los siguientes: con 
media móvil de dos periodos el pronóstico para el 2018 es de \$ 604,9 millones con un $M A D=91,3 \mathrm{mil}$, mientras que con media móvil de tres periodos el pronóstico es de $\$ 634,2$ millones con un

$M A D=107,9 \mathrm{mil}$. A continuación se presentan la serie real y las series pronosticadas.

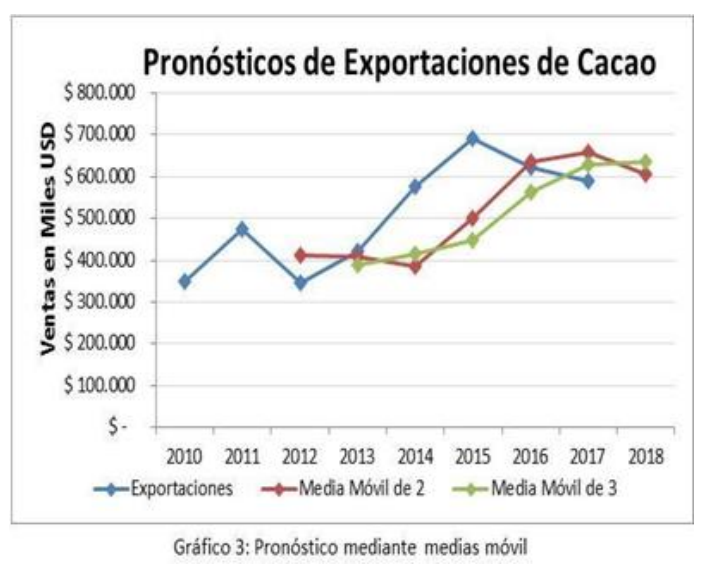

- Promedios Móviles Ponderados: Con el uso del método de media móvil ponderada de dos periodos con 0.8 y 0.2 como ponderaciones el pronóstico fue de $\$ 595,1$ millones para el 2018 con un $M A D=94,6 \mathrm{mil}$, mientras que con una media móvil de tres periodos con 0.7 , 0.2 y 0.1 como ponderaciones, el pronóstico para el 2018 fue de $\$ 605,4$ millones con un $M A D=$

89,9 mil, a continuación, se presenta la serie real conjuntamente con las pronosticadas.

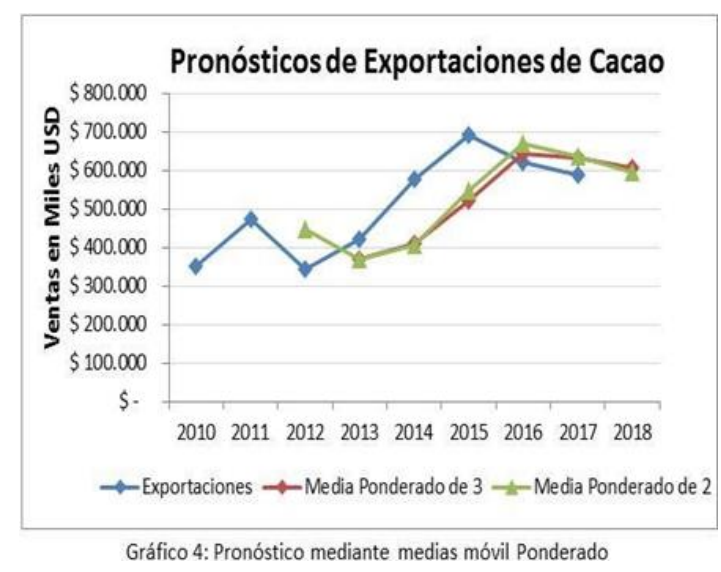

- Suavización Exponencial Simple: Con la finalidad de identificar el mejor método para realizar el pronóstico más acertado, ahora aplicamos el método de suavización simple. Para identificar el valor de $\alpha$ óptimo utilizamos la ayuda del Solver de Excel para minimizar el cuadro del error medio (MSE) de acuerdo al siguiente modelo:

$$
\text { Minimizar } Z=M S E=\frac{\sum E 2 t}{n}
$$

Sujeto a las siguientes restricciones:

$$
0 \leq \alpha \leq 1
$$


El valor de $\alpha=0.96$ hace que el MSE sea el menor, el pronóstico para las exportaciones en el 2018 es de $\$ 589,9$ millones con un $M A D=96,3$ mil asociado.

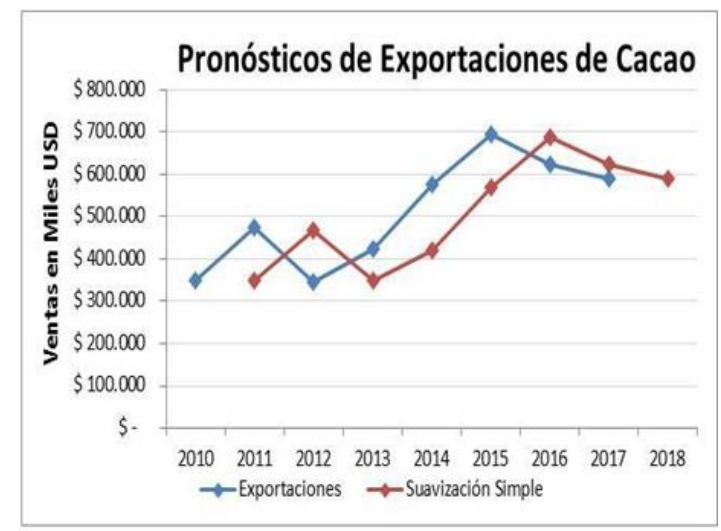

Gráfico 5: Pronóstico mediante suavización exponencial simple

- Suavización Exponencial Ajustada a la Tendencia: Analizando la serie de exportaciones de cacao, podemos ver que existe un periodo de incremento en las exportaciones entre el 2012 y 2015, para luego entrar en un periodo de decrecimiento. El siguiente método aplicado para estimar las exportaciones toma en cuenta estas tendencias en la serie. Al igual que en el método de exponencial simple utilizamos la ayuda del Solver de Excel para encontrar los parámetros $\alpha$ y $\beta$ óptimos.

$$
\text { Minimizar } Z=M S E=\frac{\sum E 2 t}{n}
$$

Sujeto a las siguientes restricciones:

$$
\begin{aligned}
& 0 \leq \alpha \leq 1 \\
& 0 \leq \beta \leq 1
\end{aligned}
$$

El valor de $\alpha=1$ y $\beta=0.39$ hace que el MSE sea el menor, el pronóstico para las exportaciones en el 2018 es de $\$ 599,1$ millones con un $M A D=79,3$ mil asociado.

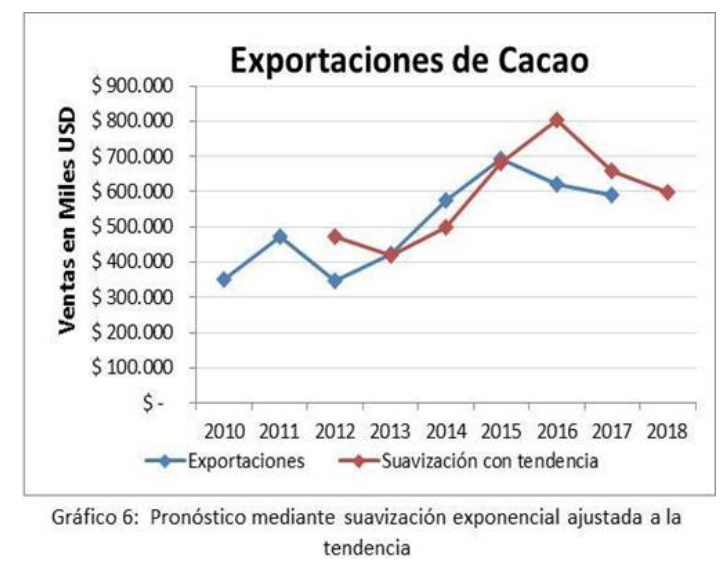

- Análisis MAD y E por cada método aplicado: Las exportaciones presentan una tendencia creciente entre el 2012 y 2015, para luego bajar los últimos dos años. Este comportamiento se ve reflejado en los métodos de suavización simple y promedio ponderado que responden 
mejor a las medidas de MAD y $E$. A pesar que suavización con tendencia tiene una MAD menor, descartamos este método ya al tener una serie con comportamientos crecientes y decrecientes, evidentemente este método no es aplicable ya que prioriza la tendencia con anterioridad.

La suavización exponencial simple presenta resultados consistentes en las dos medidas de error, por eso elegimos este método para nuestro pronóstico, de esta manera el pronóstico de exportaciones para el 2018 es de $\$ \mathbf{5 8 9 , 9}$ millones.

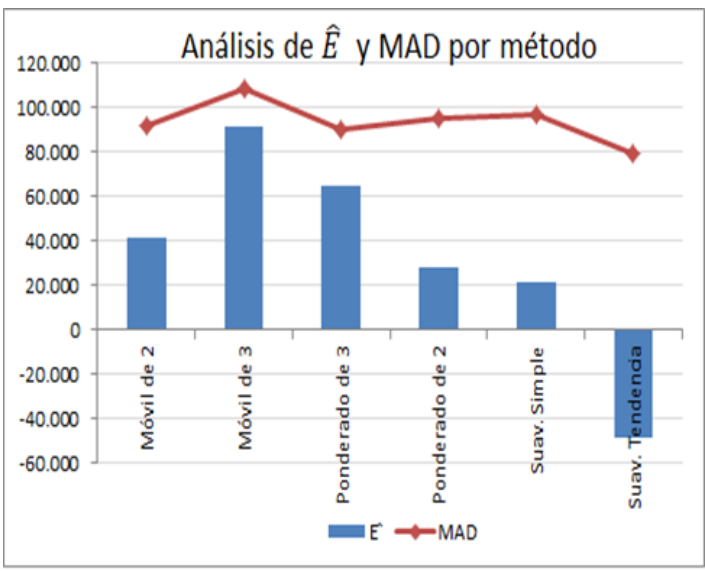

Gráfico 7: Errores de Pronóstico Promedio

- Exportaciones Trimestrales: La siguiente gráfica nos muestra las exportaciones trimestrales de los dos últimos años, en ella se puede observar que las exportaciones son más altas en el primer y cuarto trimestre, mientras que registra un decrecimiento en el segundo y tercer trimestre del año. Si las exportaciones pronosticadas para el 2018 son de $\$ 589,9$ millones de dólares, vamos a estimar las exportaciones para los cuatro trimestres utilizando el método multiplicativo.

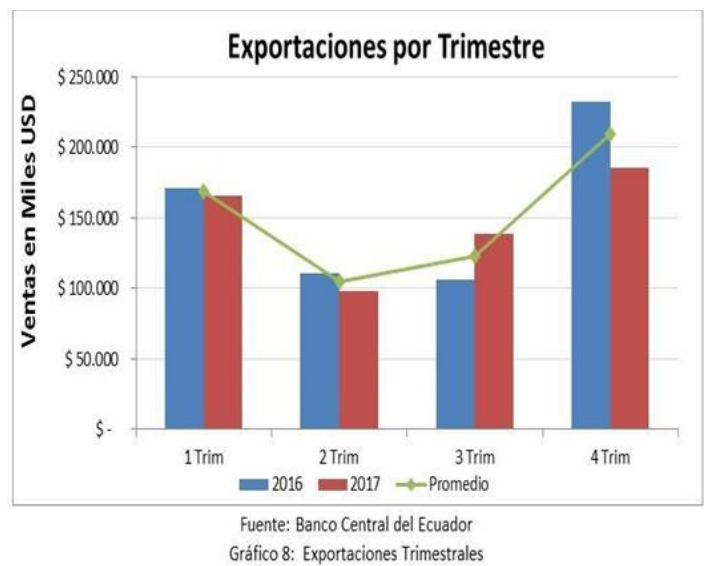

Primero obtenemos los índices estacionales para cada trimestre y luego calculamos un promedio por cada uno de los periodos. La siguiente tabla muestra los índices estacionales promedio junto con los índices por año, como se puede observar el mayor peso lo tienen el primer y cuarto trimestre, ya que ellos tienen las exportaciones más altas durante el año. 
Tabla 1: Índices Estacionales por trimestre

\begin{tabular}{lccr}
\hline Trimestre & $\mathbf{2 0 1 6}$ & $\mathbf{2 0 1 7}$ & Promedio trimestral \\
\hline 1 Trim & 1.10 & 1.13 & 1.12 \\
$\mathbf{2}$ Trim & 0.72 & 0.67 & 0.69 \\
3 Trim & 0.69 & 0.94 & 0.81 \\
4 Trim & 1.50 & 1.26 & 1.38 \\
Total & $\mathbf{4 . 0 0}$ & $\mathbf{4 . 0 0}$ & $\mathbf{4 . 0 0}$ \\
\hline
\end{tabular}

Finalmente calculamos el pronóstico de las exportaciones por trimestre para el año 2018, para ello multiplicando el promedio de los índices estacionales de cada trimestre por el valor pronosticado para el 2018, pero este valor lo dividimos para el número de periodos (cuatro trimestres).

Tabla 2: Pronóstico 2018 por trimestre

\begin{tabular}{lr}
\hline Trimestre & \multicolumn{1}{c}{ Pronóstico 2018 } \\
\hline 1 Trim & $\$ 147,4 \times 1.12=\$ 164,5$ Millones \\
2 Trim & $\$ 147,4 \times 0.69=\$ 101,9$ Millones \\
3 Trim & $\$ 147,4 \times 0.81=\$ 120,0$ Millones \\
4 Trim & $\$ 147,4 \times 1.38=\$ 203,5$ Millones \\
Total & $=\$ 589,9$ Millones \\
\hline
\end{tabular}

El siguiente gráfico nos muestra el comportamiento de las exportaciones históricas de los dos últimos años y el pronóstico para el 2018 de manera trimestral.

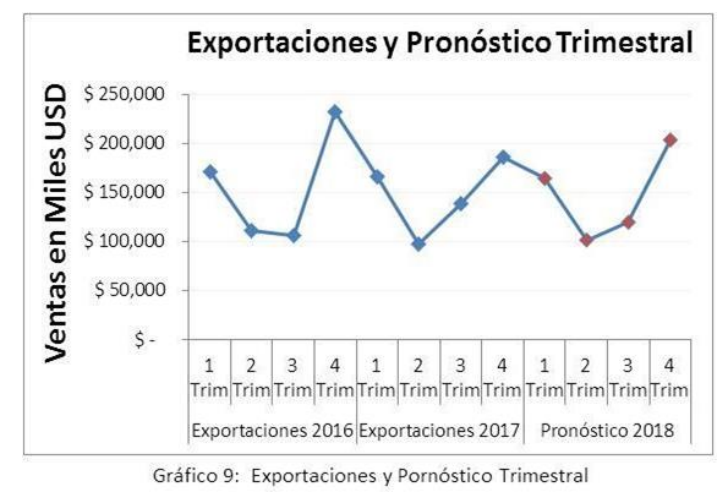

\section{Conclusiones}

Los resultados obtenidos en el presente trabajo pronostican $\$ 589,9$ millones de dólares en exportaciones por cacao para el 2018, un incremento del $0.3 \%$ con relación a lo registrado en el 2017 , en el cual se exportaron $\$ 588,4$ millones.

Esta proyección está sujeta a muchos factores entre ellos al cambio del precio en el producto. Cabe mencionar que en el 2017 los precios cayeron un 50\% con relación a lo registrado en el 2016, de seguir con esta tendencia los pronósticos para la exportación podrían ser mucho menor a lo pronosticado. 
Otros factores que influyen directamente en el pronóstico es el cambio del clima y las plagas, estos factores pueden reducir la producción en las fincas e incrementar los costos de producción para combatir las plagas.

Los métodos que se aplicaron fueron: medias móviles, medias móviles ponderadas, suavización exponencial simple y suavización exponencial con tendencia. Los resultados variaron dependiendo del modelo, siendo el modelo de suavización exponencial simple el que mejor se ajustó a la serie real. El estudio de estacionalidad que realizamos a las exportaciones en los dos últimos años nos permitió realizar el pronóstico trimestral para el 2018 utilizando el método multiplicativo.

Con la ayuda del Solver de Excel se puedo estimar los factores de suavización para los modelos exponencial simple y exponencial doble. Los métodos aplicados en el presente trabajo ofrecen dos características muy importantes que se desea en los modelos matemáticos: una solución fácil de implementar en un computador y una interpretación sencilla de los resultados.

\section{Bibliografía}

Anderson, D., Sweeney, D., Williams, T., Camm, J., \& Martin, K. (2011). Métodos cuantitativos para los negocios. cengage learning.

BOADA, A. J. (2017). Sistema de proyección de la demanda. Caso práctico de predicción automatizada en empresas de venta por catálogo. rpe.ceipa.edu.co, 23-41.

Contreras, A., Atziry, C., Martínez, J., \& Sánchez, D. (2016). Análisis de series de tiempo en el pronóstico de la demanda de almacenamiento de productos perecederos. Estudios Gerenciales, 387-396.

Corres, G., Passoni, L., Zárate, C., \& Esteban, A. (2014). Estudio comparativo de modelos de pronóstico de ventas. Iberoamerican Journal of Industrial Engineering, Florianópolis, SC, Brasil, 113-134.

ENRÍQUEZ, J. H. (2009). Pronósticos de Ventas para la Administración Estratégica de los Recursos en una Empresa del Sector Automotriz-Edición Única. Instituto Tecnológico y de Estudios Superiores de Monterrey.

Eppen, Gould, Schmidt, Moore, \& Weatherford. (2000). Investigación de Operaciones para la Ciencia Administrativa. PEARSON.

http://www.ecuadorinmediato.com. (17 de 01 de 2018). http://www.ecuadorinmediato.com. Obtenido de http://www.ecuadorinmediato.com: http://www.ecuadorinmediato.com/index.php?module=Noticias \&func=news_user_view \&id=2818831354

http://www.revistalideres.ec. (s.f.). Obtenido de http://www.revistalideres.ec: 
http://www.revistalideres.ec/lideres/cacao-ecuatoriano-historia-empezo-siglo.html

KRAJEWSKI, L., RITZMAN, L., \& MALHOTRA, M. (2008). Administración de Operaciones. México: Pearson Prentice Hall.

MURILLO, J., TREJOS, A., \& CARVAJAL, P. (2003). Estudio del pronóstico de la demanda de energia electrica, utilizando modelos de series de tiempo. Scientia et Technica, 37-42.

Valencia, A., Lozano, C., \& Moreno, C. (2007). Modelo de promedios móviles para el pronóstico horario de potencia y energía eléctrica. El Hombre y la Máquina Universidad Autónoma de Occidente Colombia, 96-105.

Zuluaga, A., Molina, P., \& Guisao, É. (2011). La planeación de la demanda como requisito para la gestión de las cadenas de suministro en las empresas en Colombia. Revista Politécnica. 